\title{
Vulnérabilité de l'organisation des interactions institutionnelles face aux écrans : le cas singulier du traitement d'un appel visiophonique au tribunal
}

\author{
Veyrier, Clair-Antoine \\ Télécom ParisTech \\ clair-antoine.veyrier@telecom-paristech.fr \\ Licoppe, Christian \\ Télécom ParisTech \\ christian.licoppe@telecom-paristech.fr
}

\section{Introduction}

Les dispositifs de vidéocommunication et leurs contextes d'utilisations se sont considérablement diversifiés. Ils ne se limitent pas à un usage personnel (de Fornel, 1994 ; Rintel, 2012), mais se déploient dans des environnements professionnels telle la téléchirurgie (Mondada, 2004), ou institutionnels comme la prison (Bonu, in press). Mondada (2007) montre l'effet configurant du dispositif de visioconférence sur l'organisation de l'interaction en se focalisant plus particulièrement sur les ouvertures. La connexion visiophonique se produit sur le modèle de l'appel où l'une des deux parties initie la connexion avec le site distant. Schegloff $(1968,1973,1986)$ a pu montrer, concernant les appels téléphoniques, comment les membres s'orientent vers une paire relationnelle standardisée (Sacks, 1972) pour organiser la parole. Licoppe et Tuncer (in press) traitent l'irruption d'un appel distant dans la perspective de la gestion de la multiactivité. L'orientation vers le traitement de la sonnerie en tant que sommation se produit graduellement et collaborativement à travers des actions successives verbales et incarnées. L'utilisation « d'écrans connectés » rend plus vulnérable le déroulement séquentiel des activités en coprésence. Leur dimension disruptive altère potentiellement la progressivité de l'activité.

Dans le contexte judiciaire, Atkinson \& Drew (1979) ont pu montrer le système d'organisation spécifique des tours de paroles dans les procès. Cette dimension institutionnelle de l'organisation de la parole (Drew \& Heritage, 1992) permet de maintenir l'intelligibilité de ce qui se produit à la cour comme des actions reconnaissables pour le justiciable. Le président de séance s'oriente vers le maintien de cette intelligibilité en organisant la parole et en veillant aux droits participatifs des membres. Dès lors, la gestion de ce déroulement et le contrôle de cette progressivité sont un enjeu pour l'activité judiciaire.

L'usage de la visioconférence au tribunal a considérablement augmenté dans le système judiciaire français. C'est le cas en particulier des pré-procès où le justiciable fait appel d'une détention provisoire. Pour réduire les coûts, le prévenu comparaît depuis la prison par vidéoconférence devant la chambre de l'instruction. La chambre décide de les maintenir incarcérés ou de les libérer selon différentes modalités en attendant leur procès. Elle se réunit sur une base hebdomadaire pour traiter une dizaine de dossiers successivement. L'introduction de la visioconférence dans la chambre d'instruction modifie quelque peu la transition entre deux dossiers. Les juges peuvent alterner des affaires avec un prévenu " en présence » avec d'autres par vidéoconférence. L'enchaînement des dossiers de différentes maisons d'arrêt ne permet pas une préparation du dispositif de visioconférence en amont de la séance une fois pour toutes. La connexion visiophonique avec une prison doit être réalisée pendant la transition entre deux dossiers. C'est la cour qui gère les appels visiophoniques avec les prisons afin de maintenir un contrôle sur l'organisation de l'audience. 
Nous souhaitons montrer dans cet article que l'amorce d'un appel produit des attentes normatives pour l'appelant. Elle présuppose en particulier une forme de disponibilité. Ainsi, la gestion d'une multiactivité dans la production d'un appel nécessite une coopération avec les membres en présence. De ce fait, ces attentes normatives ne sont pas sans incidence sur le déroulement des audiences. Elles introduisent une forme de vulnérabilité au traitement de ces apparitions.

\section{Approche et méthode}

L'étude s'appuie sur un travail de terrain dans les cours d'appel de deux villes françaises. Dans une approche vidéo-ethnographique, nous avons documenté les pratiques de la chambre de l'instruction lors de pré-procès qu'ils soient en coprésence ou par vidéocommunication. L'enregistrement audiovisuel des audiences permet d'appréhender non seulement les pratiques verbales, mais aussi incarnées dans une approche multimodale de l'interaction.

Schegloff (1987) distingue deux méthodes en analyse de conversation. La première est l'établissement de collections de phénomènes pour appréhender les procédures systématiques mises en œuvre par les membres pour un même type d'action. Le travail sur les ouvertures téléphoniques (Schegloff, 1967) est de ce point de vue exemplaire. Il a permis de démontrer les procédures systématiques des membres pour commencer un échange téléphonique. La seconde approche est celle de l'analyse d'un cas singulier. L'analyse d'un appel d'urgence qui a mal tourné (Whalen, Zimmerman, \& Whalen, 1988) mobilise cette seconde approche. Il ne s'agit plus de démontrer un procédé systématique, mais la trajectoire complexe de cet appel d'urgence atypique en s'appuyant sur des recherches antérieures. L'analyse du cas singulier permet aux auteurs de rendre compte de la multiplicité des dysfonctionnements interactionnels et des effets possibles. Nous adopterons ici l'analyse d'un cas singulier.

Il s'agit d'un épisode «atypique » dans lequel une perturbation technologique (une difficulté à établir la connexion) modifie l'organisation séquentielle de l'audience. Le président de la cour a décidé de changer l'ordre des dossiers et de commencer l'audience d'un autre prévenu dans la même affaire. Pendant cette nouvelle audience, le juge assesseur continue d'essayer d'établir la connexion avec la maison d'arrêt. Notre analyse se focalisera sur le traitement de l'appel « réussi » qui se produit alors qu'un avocat est en train de plaider. Le traitement de cet appel en pleine audience en cours révèle les attentes normatives induites par l'appel visiophonique.

Figure 1 Configuration spatiale de la cour et notation des participants pour la transcription

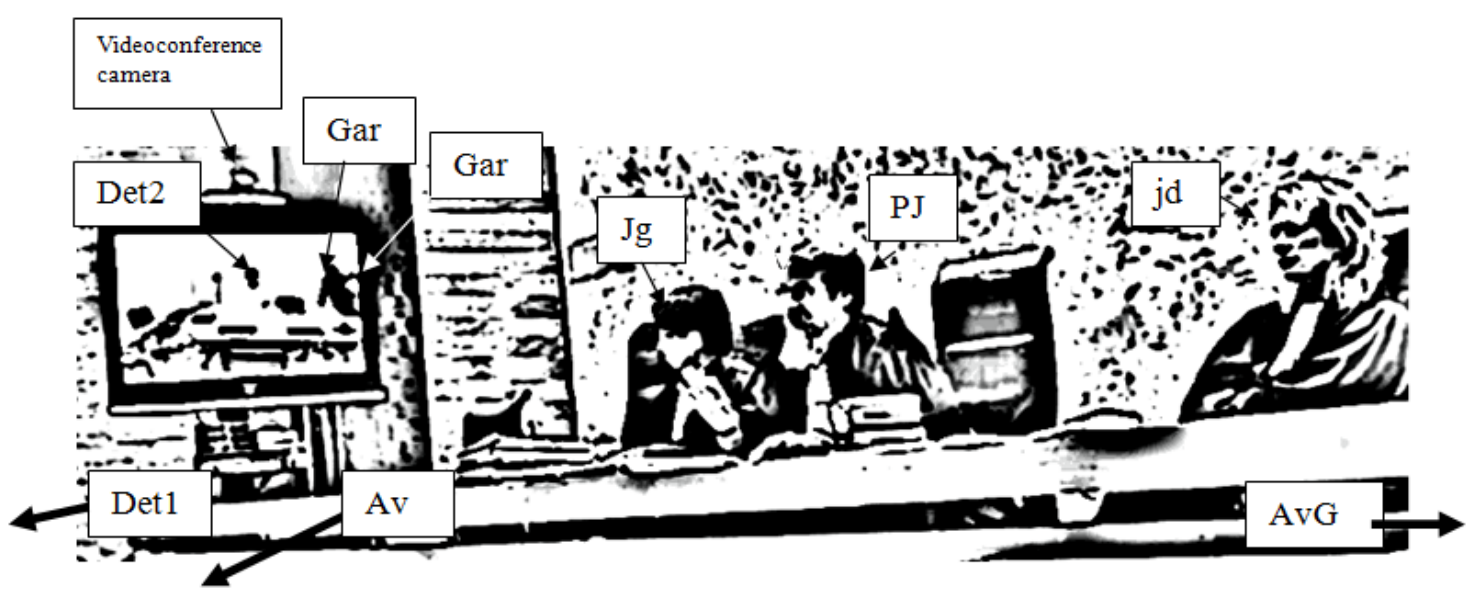

L'audience a été filmée (cf. Figure 1) et transcrite selon le système jeffersonnien (Jefferson, 2004) aménagé pour inclure la multimodalité (ICOR, 2007). La caméra permet de saisir à la fois le dispositif de visioconférence disposé sur le côté de la cour ainsi que l'activité des trois juges : le président de la cour, noté PJ, et ses deux assesseurs JD et JG. 
Figure 2 Écran de visioconférence partagé (splitscreen)

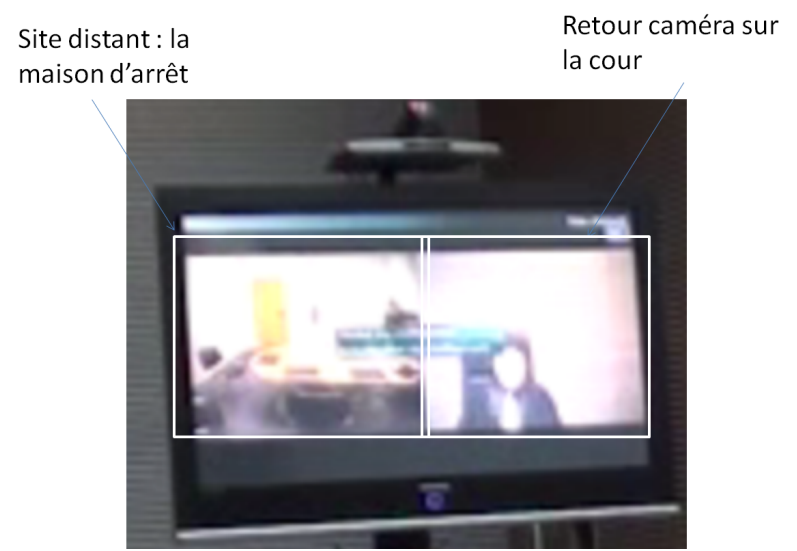

Le juge assesseur le plus proche du dispositif, JG, a la charge de gérer la visioconférence à l'aide d'une télécommande. Cette dernière lui permet entre autres d'amorcer un appel avec une maison d'arrêt, de modifier l'orientation de la caméra placée au-dessus de l'écran (cf. Figure 1) ou de faire apparaître le retour visuel de la caméra.

\section{Produire un appel visiophonique en cours d'audience}

\subsection{Perturbation technologique : entre attente de l'appel et progressivité de l'ordre du jour}

Lors d'une même session, la chambre de l'instruction traite successivement différents dossiers. Dans l'épisode que nous examinerons, la cour vient de se déconnecter avec une première maison d'arrêt et s'apprête à se connecter avec un autre site pour traiter un nouveau dossier, celui du prévenu noté Det1. Cependant, les premières tentatives d'appels échouent à cause d'un changement de salle et de numéro de visioconférence à la maison d'arrêt. Cet évènement va entrainer des difficultés de connexion pour la cour. Il va s'en suivre une série de tentatives et d'échanges téléphoniques entre le greffier et la maison d'arrêt pour initier la connexion pendant une dizaine de minutes. Le prévenu Det2 attendu pour l'audience suivante arrive dans la salle d'audience (Extrait 1). L'avocat est déjà là car il plaide à la fois pour Det1 et Det2. À ce moment, le président s'oriente vers un changement d'organisation de l'ordre des audiences. L'énoncé « et on est à vous » (1.2) est ambigu, il laisse penser que la cour va traiter le dossier de Det2 de manière imminente.

Extrait 1
1. Pj
asseyez-vous monsieur $((\operatorname{det} 2))$ on est en train de
2 .
régler un problème technique et on est à vous

Quelques minutes plus tard, dans l'extrait 2a, la greffière termine une conversation téléphonique (1.1-2) avec la maison d'arrêt pour traiter le problème de connexion visiophonique. Après avoir vérifié le numéro d'appel avec la maison d'arrêt, la greffière s'oriente vers la clôture de l'appel téléphonique (1.1-2). Le juge assesseur amorce à nouveau l'appel visiophonique (1.2) sans succès comme le relève le président (1.5). Selon Turner (1972), « une condition pour que l'occasion sociale se réalise en tant qu'occasion, est « le rassemblement préalable des participants » (1972: 370). L'échec provisoire de la connexion ne permet pas de commencer l'audience avec Det1 à distance. Néanmoins, la présence préalable de Det2 et de son avocat sont des conditions réunies. La décision du président « on va commencer quand même » (1.7) est ainsi motivée par l'échec de l'appel («y’a pas de réponse » (1.5)) et la présence du prévenu suivant («puisque Det2 est là » (1.8)). Cette décision traduit une double orientation : celle de maintenir 
une progressivité dans le déroulement attendu de la séance et l'obligation de traiter tous les dossiers prévu à l'ordre du jour. En effet, l'énoncé « et on essaiera de se connecter» (1.7-8) va être traité comme une instruction par le juge assesseur JG en charge de la visioconférence.

Extrait 2a
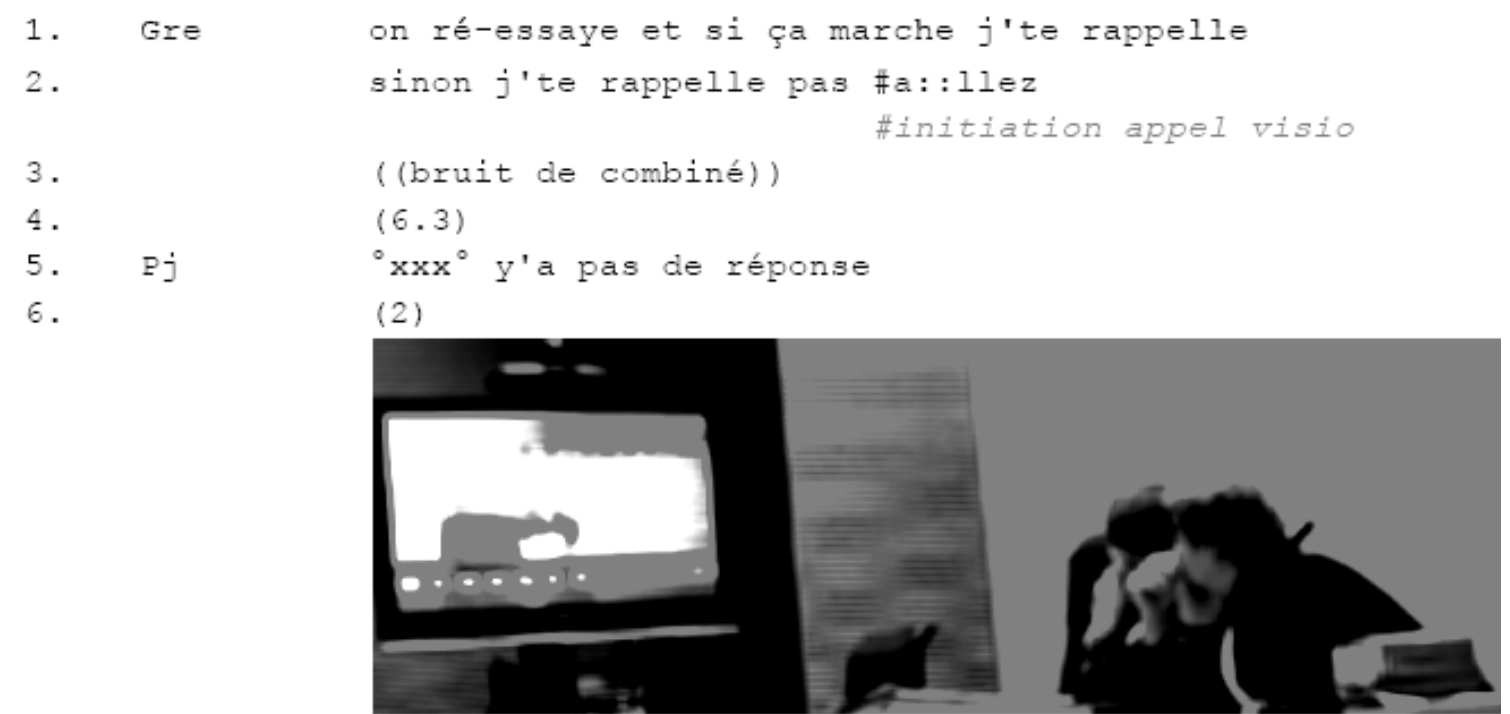

7 .

Son va commencer quand même heu: : et on essaiera

8.
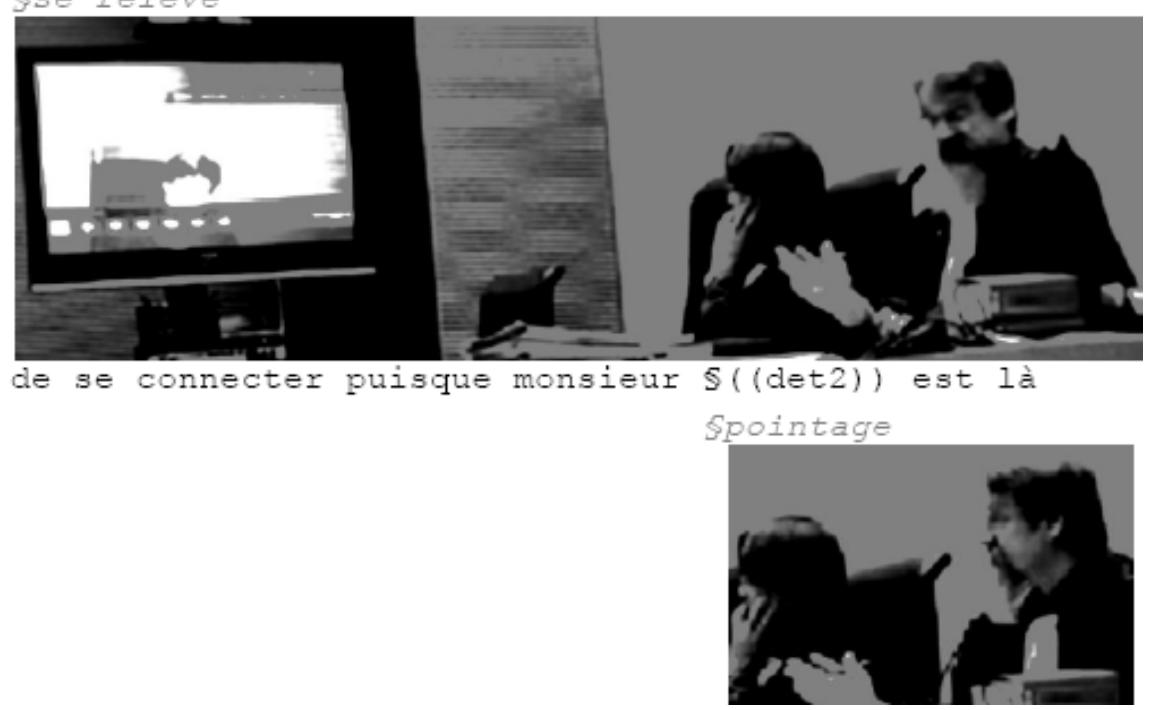

Alors que le second juge assesseur JD commence (1.15) (extrait 2b) la présentation du résumé, le second juge assesseur va poursuivre ses tentatives de connexion pendant une quinzaine de minutes environ. Dans ce contexte, les tentatives d'appels introduisent une incertitude dans la progressivité de l'audience par la possibilité qu'une tentative d'appel visiophonique aboutisse. Cette multiactivité peut avoir des implications séquentielles quant au déroulement de l'audience. 


\section{Extrait $2 b$}

9.
10.
11.

(0.6) on va faire une fois le:

(1.5)

Sle rapport

12. AvG

\$regard vers Av

$\$ p j$

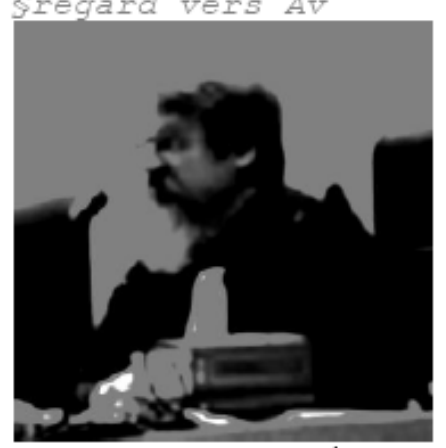

vous pourrez faire \$de: : s commentaires

sse tourne vers jd

13.

intéressants sur le l'outil

14.

$$
8(1.5)
$$

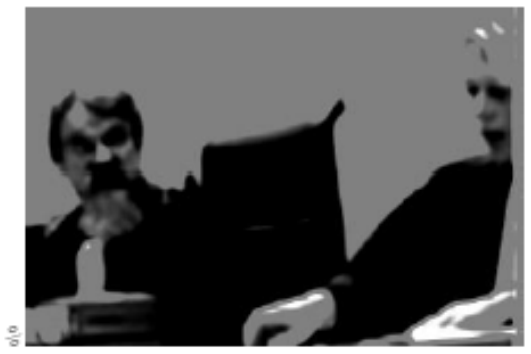

bon alors: : heu (1.5) monsieur (2) ((det2))

\subsection{Plaidoirie et tentatives de connexion : gestion de la multiactivité}

Au bout d'une quinzaine de minutes d'audience, la dernière tentative de connexion avec la maison d'arrêt aboutira. Un résumé de l'affaire a déjà été présenté, le prévenu en présentiel a été entendu et l'avocat a déjà commencé sa plaidoirie en faveur de son client en présence depuis six minutes environ, lorsque l'appel aboutit. La production d'un appel visiophonique à un placement séquentiel inhabituel (en pleine audience) constitue l'unique occurrence du corpus observé. Ce cas atypique permet d'analyser la gestion distribuée entre les membres d'une multiactivité induite par la production de l'appel. La gestion de la multiactivité a été traitée dans la perspective des appelés (Licoppe \& Tuncer, in press), mais pas encore dans celle de l'appelant. La production d'un appel téléphonique ou visiophonique présuppose une disponibilité lorsque ce dernier aboutit. 
Les extraits (3a) à (3e) sont la transcription de la même séquence que nous allons analyser en détail dans la suite de cet article. Dans cet épisode, le juge assesseur (JG) doit gérer deux activités : suivre l'audience et initier la connexion au site distant. L'avocat (Av) plaide depuis six minutes environ au début de l'Extrait 3. Le juge assesseur JG mobilise une alternance du regard (1.1-6) entre l'avocat (Av) et l'écran pour gérer visiblement cette multiactivité et maintenir le cadre de participation.

Extrait $3 \mathrm{a}$

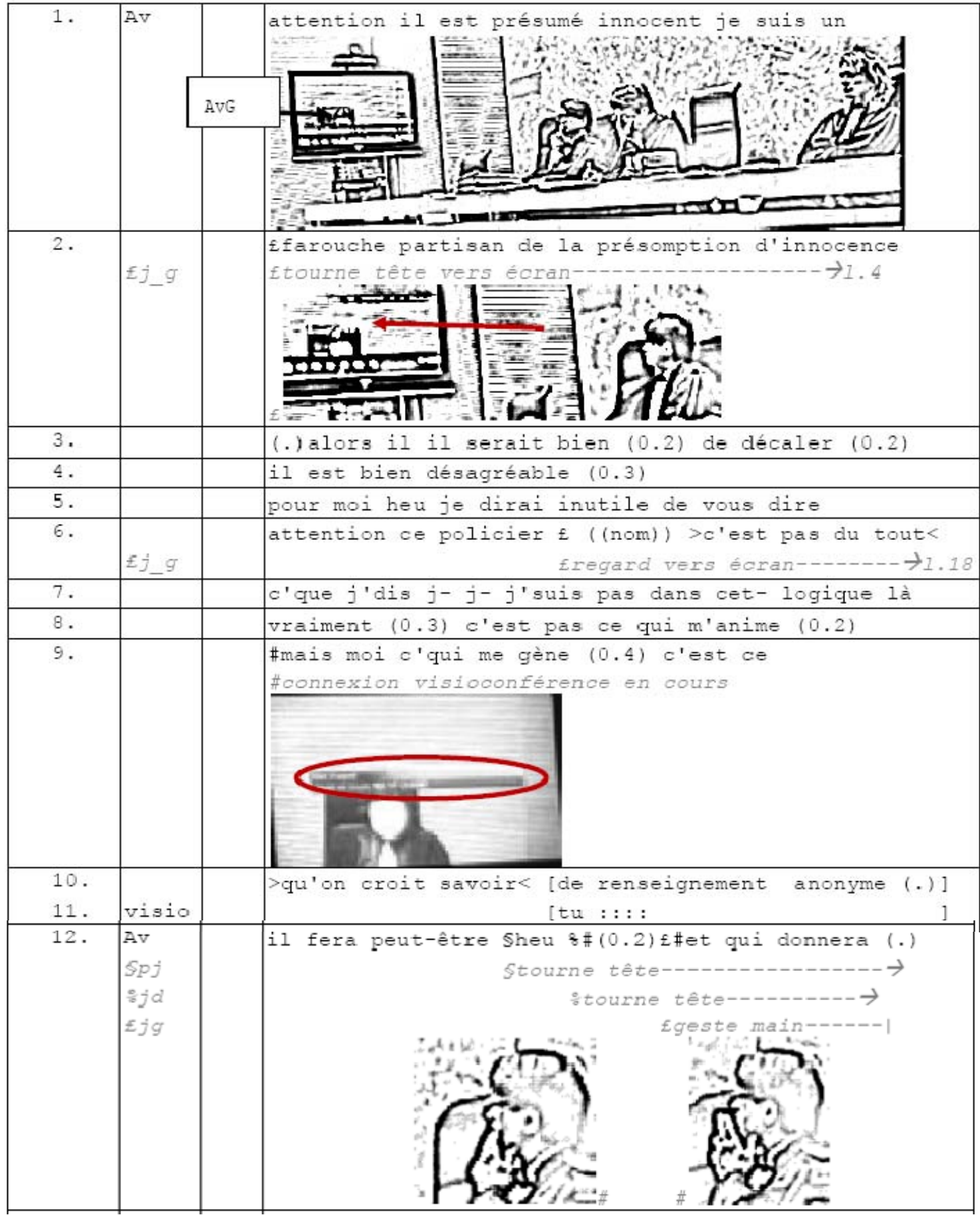




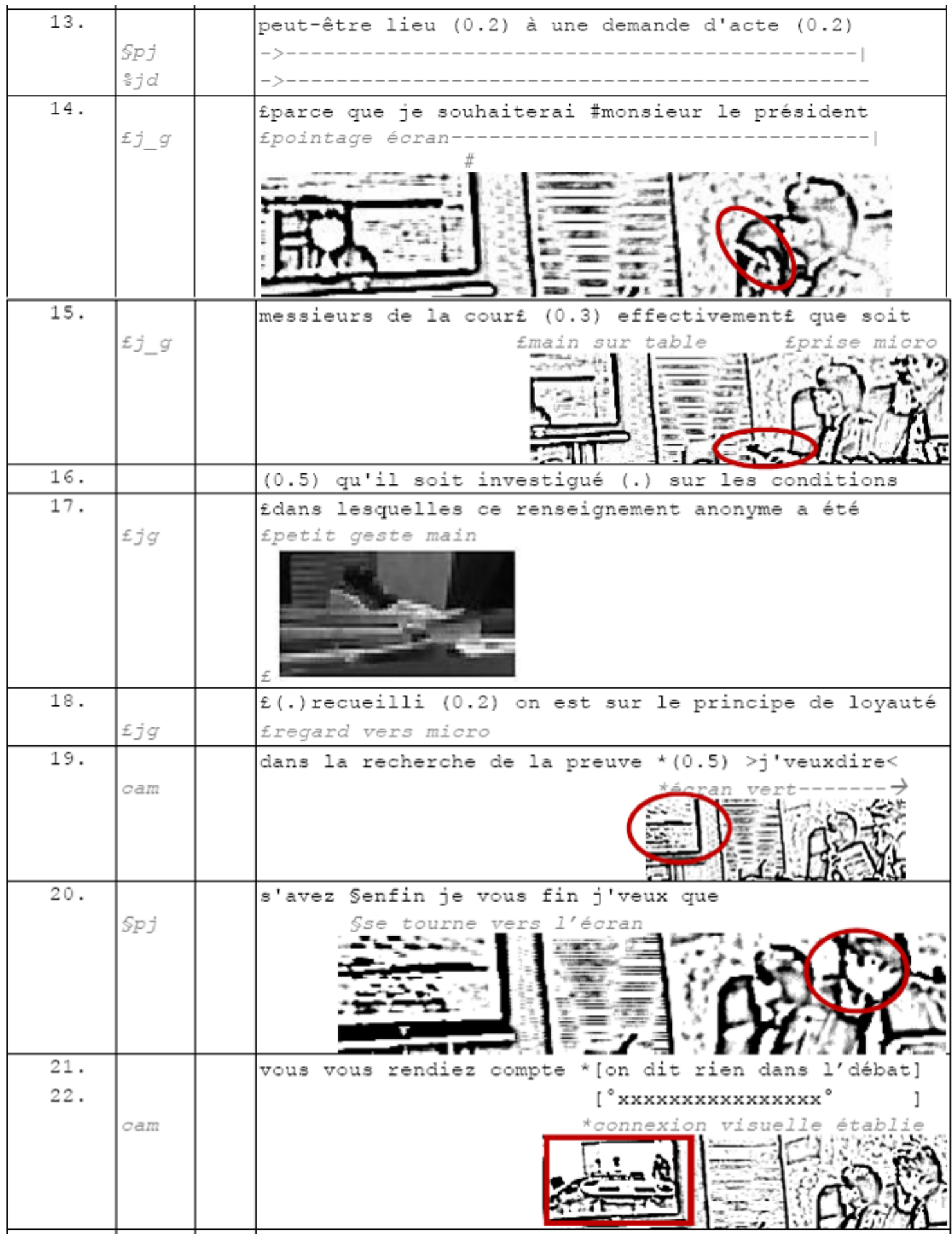

Le juge assesseur (JG) suspend l'alternance de son regard lorsqu'une notification visuelle d'appel en cours (1.9) apparaît à l'écran. La notification visuelle est suivie d'une notification sonore (1.11) qui sera traitée visuellement a minima par un regard du président $(\mathrm{Pj})$ et du second juge assesseur (JD) (1.12) sans perturber la progressivité de la plaidoirie de l'avocat. Le juge assesseur (JG) produit un premier geste de la main (1.12) qui peut s'apparenter à un pointage. Celui-ci n'est pas traité par les autres membres. Un 
second pointage vers l'écran (1.14) est suivi séquentiellement par la saisie (1.15) du microphone. Il s'agit d'une action préparatoire qui projette l'imminence d'une connexion avec le site distant et de son traitement. Un troisième geste (1.17) est accompagné d'une moue de la tête. On peut faire l'hypothèse que ce dernier geste s'adresse à l'huissier qui est en charge des relations téléphoniques avec les maisons d'arrêt. Le geste de la main produit une description visible (account) de l'état en cours de son activité de tentative de connexion. Le juge assesseur manipule le microphone en vérifiant son état (1.18). Le changement d'apparence de l'écran qui devient vert (1.19) est notifié par le juge assesseur qui conserve son foyer d'attention sur l'écran, mais aussi par le juge (1.20-22). Ce regard vers l'écran coordonné peut rendre compte soit d'une forme d'attention périphérique du juge, soit d'une activité du public qui attire son attention vers l'écran.

Ainsi, l'imminence de la connexion se traduit par un changement de focalisation et un désengagement relatif vis-à-vis de la cour. Le président maintient son attention vers l'avocat, mais manifeste une attention périphérique concernant la visiocommunication. La gestion des deux cours d'action par le juge assesseur implique un accomplissement multimodal.

\section{Apparition à l'écran et distribution de l'activité}

La connexion avec la maison d'arrêt est pleinement établie (1.21-22). L'aboutissement de la connexion offre une opportunité pour une transition de l'activité de plaidoirie. La connexion est visuellement notifiée par le juge assesseur, le président, ainsi que les membres distants. L'arrivée de nouveaux membres dans la salle du tribunal alors qu'une audience est en cours n'est pas rare. Les avocats ou le public peuvent arriver en avance alors que l'audience précédente n'est pas terminée. Ces va-et-vient sont visibles pour les membres, mais ne font pas l'objet d'un traitement particulier. Leur entrée silencieuse atteste d'une orientation vers l'activité institutionnelle en cours. Il en est de même dans le cas des réunions par webconférence où les membres en retard gardent le silence lorsqu'ils se connectent (Veyrier, 2012). On peut se demander comment va être traitée cette apparition de l'image et du son des sites distants par les différents membres de la cour et de la maison d'arrêt. La plaidoirie est un épisode reconnaissable de ce type d'interaction institutionnel où l'alternance des tours de parole est suspendue. L'apparition médiatisée à ce placement séquentiel particulier laisse plusieurs alternatives : maintenir l'ordre attendu de la plaidoirie ou traiter cette apparition à l'écran.

\subsection{Traitement de l'apparition par le locuteur en action et le président}

L'apparition à l'image est accompagnée de bruits de voix (1.22) en chevauchement avec la fin d'une unité de tour produite par l'avocat (1.21).

Extrait $3 b$

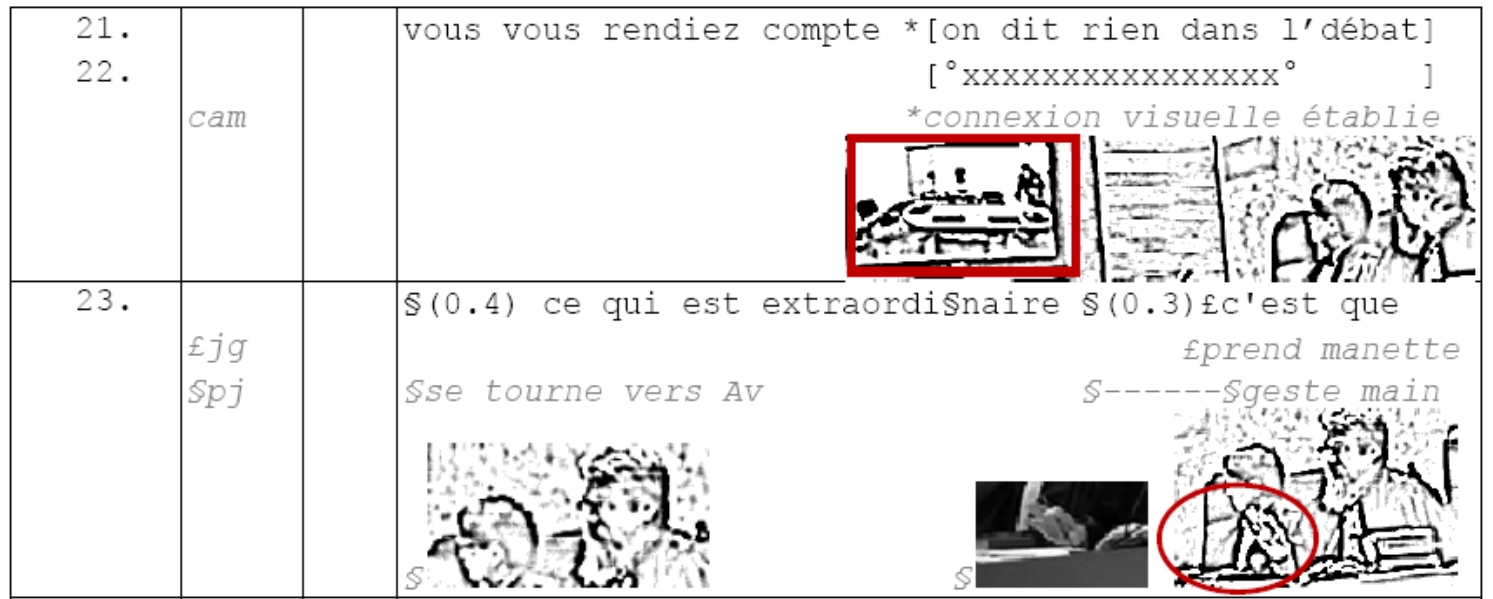


La fin d'une unité de tour ainsi que la pause (1.23) rendait possible un traitement de l'apparition du site distant. Le président se tourne vers l'avocat au moment de cette pause. Néanmoins, la construction du tour de l'avocat par une pseudo-clivée (« ce que x, c'est y ») projette ainsi une continuation de son tour de parole. Le président initie son geste de la main sur la fin de la première partie de la pseudo-clivée et l'amplifie lors de la pause (1.23). Par ce procédé gestuel graduel, le président marque son orientation vers le traitement de la connexion vidéo et une préférence pour minimiser l'interruption. L'avocat poursuit son tour par la seconde partie de la pseudo-clivée « c'est que » qui projette une complétion du tour.

Extrait 3c

\begin{tabular}{|c|c|c|c|}
\hline $\begin{array}{l}24 . \\
25 .\end{array}$ & $\begin{array}{l}\text { Garl } \\
£ j g \\
\text { Spj } \\
\therefore j d \\
\text { cam }\end{array}$ & $->$ & 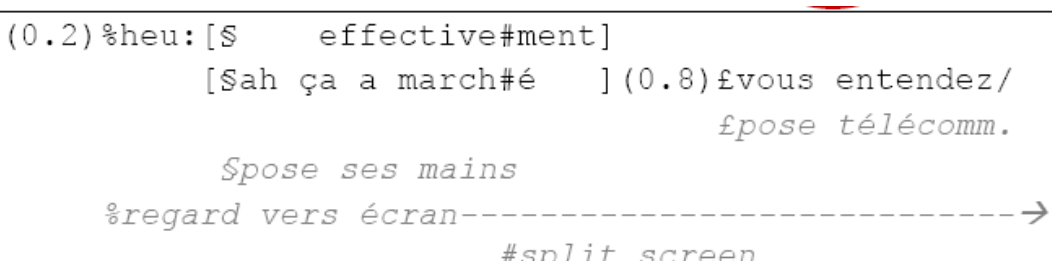 \\
\hline & & & 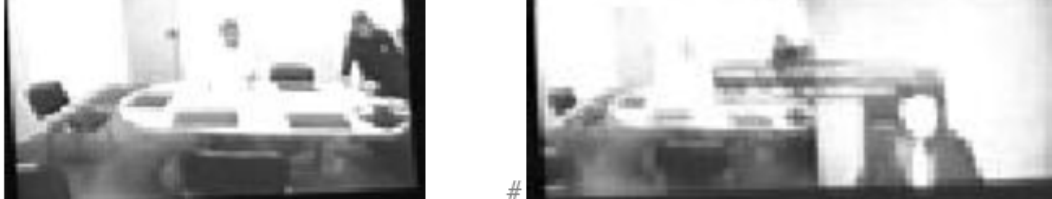 \\
\hline $\begin{array}{l}26 . \\
27 .\end{array}$ & $\begin{array}{l}? ? \\
\% j d\end{array}$ & & $\begin{array}{l}(0.9)] \\
{[((\text { sonnerie }))]} \\
->----------\mid\end{array}$ \\
\hline 28 . & $\begin{array}{l}? ? \\
\frac{\circ}{\circ} \mathrm{d} \\
£ j g\end{array}$ & & $\begin{aligned} \text { \%oui oui fmalheureusement } \\
\text { £prend télécommande } \rightarrow\end{aligned}$ \\
\hline
\end{tabular}

La pause et la particule « heu » (1.24) qui suit marquent une forme d'attention pour le geste produit par le président. Le gardien intervient en chevauchement avec l'avocat «ah ça a marché » (1.25). Cet énoncé audible à la cour conduit à une suspension du tour de parole de l'avocat. Conjointement à cet énoncé en chevauchement, le président baisse la main (1.25). La clôture de ce geste atteste la fin de la pertinence du pointage d'une perturbation devenue saillante par l'irruption de la parole du gardien dans l'espace sonore de la cour.

Le geste du juge pour interrompre l'avocat traite l'apparition à l'écran comme un évènement qui projette des droits et obligations. L'avocat ne manifeste pas la même orientation par la construction multi-unité d'un tour de parole long qui projette la poursuite de sa plaidoirie. La suspension de son tour n'est pas immédiate, mais s'appuie sur plusieurs ressources cumulées: bruits de fond, geste du président, chevauchement de la parole. Le traitement de l'apparition comme "sommation 》 est ainsi résistée par l'avocat. La tentative de reprise de la plaidoirie (1.30) (Extrait 3d) atteste ce désalignement. L'avocat traite cette apparition à distance comme celle qui se produirait en présentiel. Le geste du juge pour interrompre l'avocat n'est pas un procédé pour prendre la parole, mais pour la distribuer.

\subsection{Ajustement de l'action à l'activité en cours par le site distant}

L’énoncé « ah ça a marché » (1.25) laisse entendre que le surveillant s'oriente vers l'établissement de la connexion à ce moment-là. L'établissement d'un cadre de participation commun n'est pas immédiat. La suspension du tour de l'avocat est suivie d'une courte pause (1.25). On peut affirmer que la pause n'est pas coproduite par les deux locuteurs en action. Si l'avocat traite ce chevauchement en suspendant son tour, on peut faire l'hypothèse qu'il n'en est pas de même pour le gardien qui en l'absence de changement de locuteur après la première unité de construction de tour «ça a marché » poursuit simplement son tour. 
En premier lieu, le surveillant ne pouvait entendre l'avocat. Ce dernier n'avait aucune raison d'utiliser un microphone jusqu'à présent. Or seul l'usage d'un microphone permet d'être entendu par le site distant. En second lieu, le cadrage initial côté cour est centré sur l'avocate générale (1.1-30). Cette dernière est assise et ne parle pas. Une inspection visuelle de ce cadrage ne produit pas d'indices contextuels (Gumperz, 1982) pour inférer sur l'activité de plaidoirie. Le surveillant n'a pas d'informations préalables sur l'activité de la cour depuis le début de l'audience, car le greffier ne peut contacter la maison d'arrêt alors que la séance a déjà commencé.

Enfin, l'appel téléphonique (ou ici visiophonique) présuppose une forme de disponibilité de celui qui initie l'appel. En tant que forme d'interpellation, l'appel constitue une pré-séquence (Schegloff, 2007) qui projette d'autres actions et donc une disponibilité celui qui produit l'interpellation. La connexion avec le site distant est toujours initiée par la cour. C'est le juge qui règle et gère l'organisation des audiences et son bon déroulement. Un planning est envoyé par la cour à la maison d'arrêt pour donner un calendrier prévisionnel du déroulement des audiences. Cette organisation est donnée à titre indicatif, car la cour ne peut prévoir combien de temps dure le traitement de chaque dossier. Cette incertitude a un effet structurant dans les activités. En effet, les détenus se doivent d'être présents à l'heure du planning indiqué, mais inversement il n'est pas garanti que la cour soit à l'heure. Le surveillant traite ainsi l'appel visiophonique comme une forme de disponibilité de la cour et une activité transitionnelle. Or dans l'extrait, l'action d'appeler n'est pas l'unique activité sur le site de la cour. Il y a une tension entre une activité institutionnelle, celle de l'audience, et celle de la connexion avec le site distant.

\subsection{Le travail préparatoire du juge assesseur en charge de la visioconférence}

Le juge assesseur a anticipé l'apparition du site distant en saisissant notamment le microphone (1.15). Cette action anticipatrice laisse penser que le juge assesseur s'apprête à traiter l'apparition à l'écran par des actions verbales dès l'apparition à l'image. Or, l'apparition à l'écran (1.21-22) n'est pas suivie séquentiellement d'un geste de rapprochement devant la bouche du microphone. Ce geste préface (1.3031) une prise de parole retardée (1.36).

Le juge assesseur est orienté vers deux contraintes avant de prendre la parole : la prise en compte du placement séquentiel dans l'activité de la cour et l'orientation normative d'une mise à l'écran préalable du locuteur en action (Licoppe \& Morel, 2012). Nous avons déjà montré le travail du président pour suspendre la plaidoirie et permettre au juge assesseur de traiter l'apparition à l'écran. Nous allons examiner le deuxième aspect, le travail de cadrage préalable.

La prise du microphone est précédée par une autre activité, celle de produire un cadrage approprié. Avant l'établissement de la connexion vidéo, le cadrage initial de vidéo est centré sur l'avocate générale. Ce cadrage initial est inhabituel lors des connexions, mais n'est pas traité a priori par le juge assesseur dont l'activité principale est d'aboutir à l'établissement de la connexion. Lors de l'apparition à l'écran (1.22), l'image du site distant apparaît en plein écran. L'image retour de la caméra sur la cour n'est pas disponible et ne permet pas immédiatement un contrôle visuel. Cette configuration matérielle prétablie aura des conséquences séquentielles sur l'organisation du traitement de la connexion avec le site distant.

Lors de l'apparition du site distant, le juge assesseur commence par saisir la télécommande de la visioconférence (1.23) pour changer la disposition de l'écran (1.25) en partageant l'écran. Il pose ensuite la télécommande (1.25) marquant la complétion (provisoire) du travail de préparation de la vidéocommunication. Le juge assesseur maintient son attention vers l'écran et saisit à nouveau la télécommande (1.28) pour produire un recadrage vidéo (1.30-31) centré sur la cour (extrait 3d). Nous pouvons faire l'hypothèse que ce recadrage répare un cadrage initial inapproprié, et s'oriente vers la production d'une action particulière, celle de mettre à l'écran le locuteur projeté. Alors que l'avocat est en action (1.30), le juge assesseur met à l'écran la cour. Par la production de ce cadrage, le juge assesseur s'oriente vers un traitement de la perturbation et non un alignement avec l'activité de l'avocat. De plus le changement de cadrage est accompagné d'un rapprochement simultané du microphone du juge assesseur, projetant une prise de parole. 
Extrait 3d

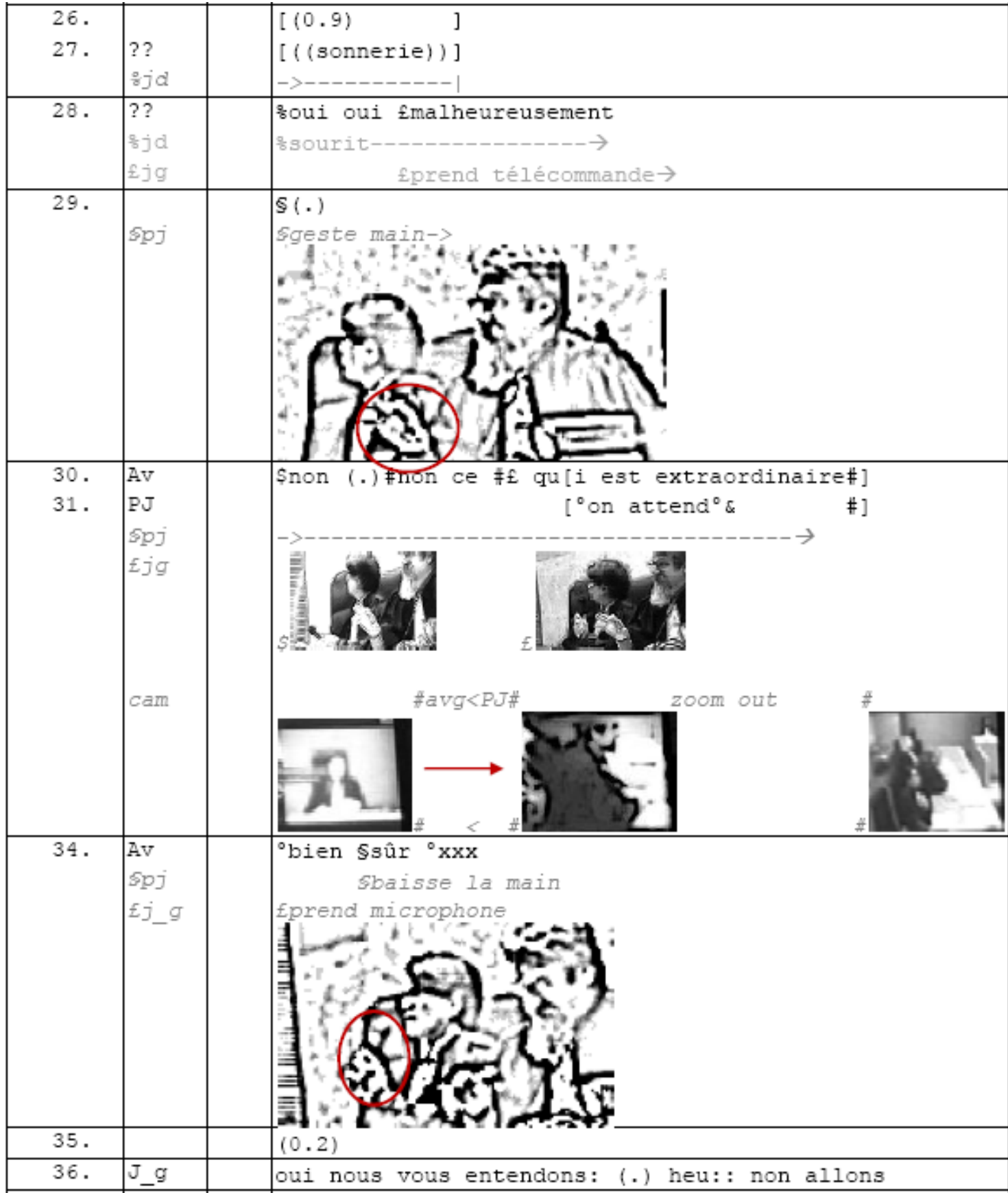

\section{Perturbation et traitement de la perturbation}

L'énoncé « vous entendez ? » (1.25) est une première partie de paire qui produit l'attente d'un second tour approprié. La complétion de la question est retardée (1.36) par des séquences insérées et multimodales (1.28-36). La production de la réponse est retardée par :

- L'ajustement multimodal du dispositif qui retarde la production d'une réponse ; 
- La nécessité d'un alignement sur l'activité pertinente en cours.

Un locuteur non identifié (probablement un avocat présent dans l'audience) produit un tour (1.28) qui prend le format d'une seconde partie de paire appropriée à la vérification (1.26). Cependant, celui-ci n'est pas audible par le site distant, car il est produit hors microphone. L'adverbe "malheureusement» qui complète la réponse positive et préférée « oui oui » (Pomerantz, 1984), modifie son sens. Il ne s'agit pas d'une réponse, mais d'un commentaire public qui traite le tour précédent comme une perturbation. Cet énoncé souligne le caractère disruptif du tour du surveillant et marque une préférence pour la progressivité de l'action. Le sourire du juge assesseur JD (1.28) atteste ce traitement. De ce point de vue, une apparition en cours d'audience projette l'attente d'une entrée silencieuse et non disruptive. La reprise de la plaidoirie par l'avocat (1.30) manifeste un alignement avec le commentaire précédent et s'oriente vers la clôture du traitement de la perturbation.

Le président anticipe le tour de l'avocat en produisant à nouveau le même geste suspensif de la main (1.29-31). Ainsi, le président atteste que le traitement de l'apparition à l'écran n'est pas clos. La pertinence conditionnelle de la production d'une réponse au surveillant (1.25) est maintenue.

La distribution de l'activité fait du juge assesseur JG le locuteur attendu pour traiter l'établissement d'une connexion avec le site distant. Ainsi, le geste de la main (1.29) du président atteste cette distribution de l'activité. Il n'a pas saisi l'opportunité de la question du surveillant pour prendre la parole, mais il attend le traitement par le juge assesseur.

L'intervention du juge assesseur est précédée par un travail de préparation du dispositif (splitscreen et recadrage sur la cour). L'avocat ne s'aligne pas avec ce travail (1.30) ce qui retarde la production d'une réponse au surveillant. Le juge assesseur maintient son microphone proche de sa bouche, mais le relâche très légèrement (1.32-33) manifestant à la fois la projection de son tour et une prise en compte de l'action en cours.

Le président ne se contente pas d'une instruction gestuelle (1.29-31) pour suspendre le tour de l'avocat, mais accompagne son geste d'une instruction verbale «on attend deux minutes » (1.31-32) suivies d'un pointage vers l'écran. On peut donc voir une amplification progressive des gestes du président: de l'émergence d'un simple geste à un couplage verbo-gestuel. L'avocat s'aligne par un acquiescement (1.33) qui marque la suspension de son tour. La seconde partie du tour du président «pour leur dire d'attendre » (1.32) permet à la fois de justifier l'interruption, et d'instruire publiquement une organisation de l'activité qui doit être relayée par le juge assesseur au site distant. La complétion de la question du surveillant est produite par le juge assesseur avec l'énoncé : « oui nous vous entendons » (1.32).

Extrait $3 \mathrm{e}$

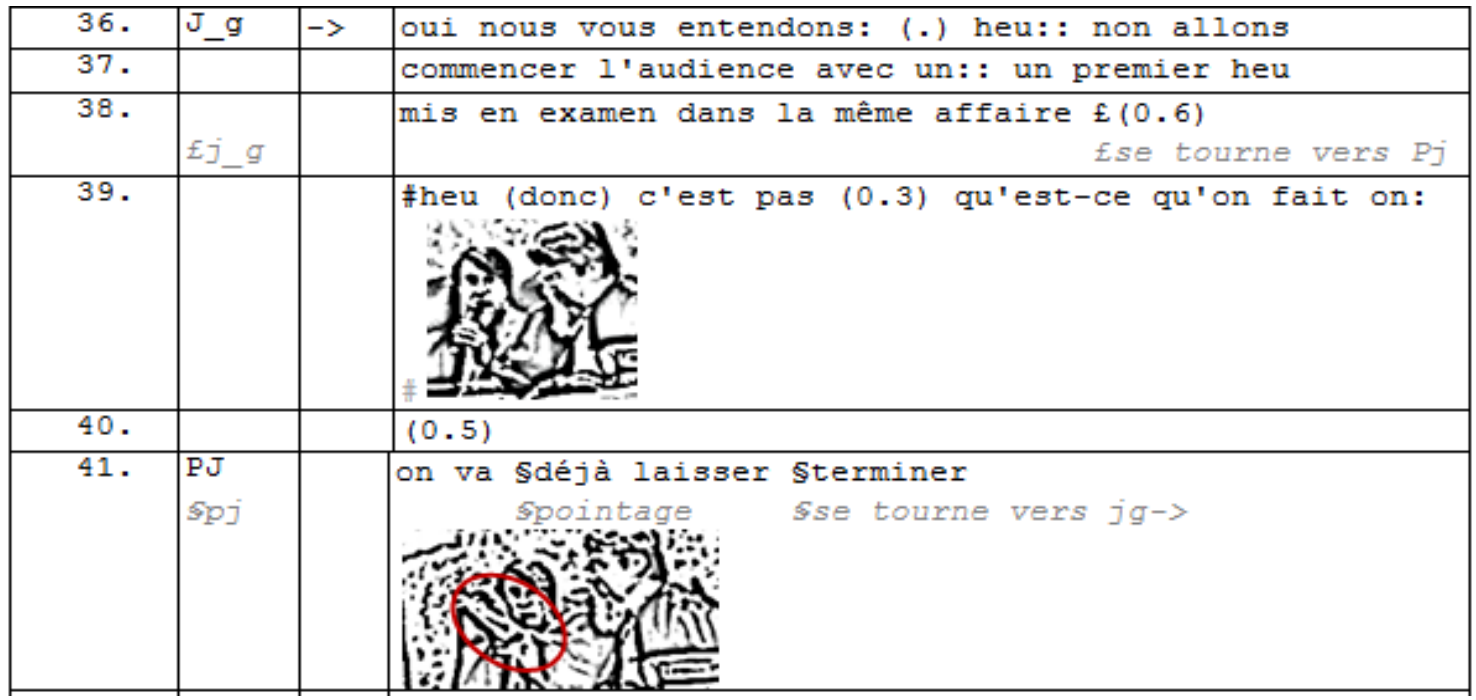




\begin{tabular}{|c|c|c|}
\hline $\begin{array}{l}42 . \\
43 .\end{array}$ & $\begin{array}{l}\text { J_g } \\
£ j g \\
\text { Spj }\end{array}$ & $\begin{array}{l}\text { [maitre ( (av))et §on lui donnera bien la parole après] } \\
\text { [£on va déjà laisser heu terminer } \\
\text { f se tourne vers écran } \\
\text {->-us] }\end{array}$ \\
\hline 44. & & smaitre $((\mathrm{Av}))$ l'avocat donc qui qui heu: train \\
\hline $\begin{array}{l}45 . \\
46 .\end{array}$ & Spj & $\begin{array}{c}\text { est en de [plaider Set ensuite heu: }(0.3)] \text { heu: } \\
\text { [((Jd talks to PJ)) } \\
\text { sse tourne vers JD }\end{array}$ \\
\hline 47. & & nous continuerons avec \\
\hline 48. & $f j \_g$ & $\begin{array}{l}\text { f움 (1.1) } \\
\text { fpose mi crophone }\end{array}$ \\
\hline 49. & $\begin{array}{l}\text { Det2 } \\
\text { gjd }\end{array}$ & $\begin{array}{l}\text { sos'accord } \\
\text { gregard vers écran-- } \rightarrow 1.57\end{array}$ \\
\hline 50. & *gar1 & $\begin{array}{l}\text { * }(0.5) \\
\text { *marche vers la porte-> }\end{array}$ \\
\hline $\begin{array}{l}51 . \\
52 .\end{array}$ & $\begin{array}{l}\text { PJ } \\
\text { Av } \\
\text { Spj } \\
\\
\text { *gar }\end{array}$ & 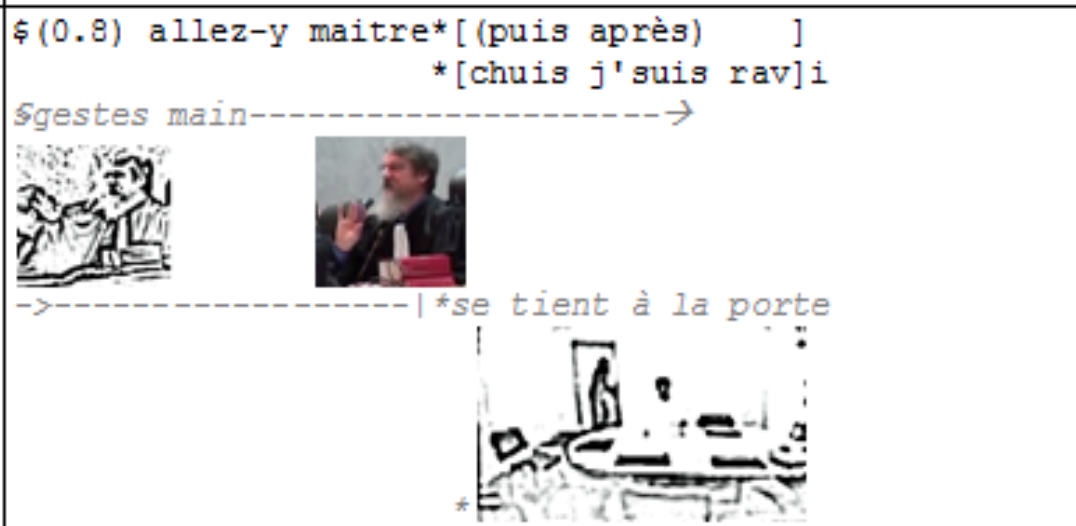 \\
\hline
\end{tabular}

L'utilisation du futur périphrastique «nous allons commencer l'audience » (1.37) est étonnante pour décrire une activité déjà commencée et toujours en cours. Nous pouvons faire trois hypothèses :

- La langue a simplement « fourchée » : « allons » à la place de « avons ».

- Le locuteur a changé la projection de son tour, mais le complète en s'appuyant sur la construction du tour déjà élaborée : il ne se réfère plus à l'audience du prévenu à distance (qui va commencer ultérieurement), mais à celle qui a déjà commencé (celle d'un "premier mit en examen dans la même affaire").

- L'utilisation du futur permet à l'assesseur de ne pas produire un compte rendu de ce qui vient de se produire en l'absence du prévenu à distance. Ce serait dans ce cas un procédé pour minimiser l'interruption de la plaidoirie de l'avocat et retarder ce compte rendu.

Le futur suggère une planification de l'organisation de l'audience par le juge assesseur. Néanmoins, par son changement d'orientation corporelle (1.36), le juge assesseur modifie le cadre de participation (Goodwin, 1979) et sélectionne le président comme destinataire tout en parlant au microphone (Lerner, 2003). Il rend public son statut épistémique vis-à-vis de la planification des actions. La proposition interrogative «qu'est-ce qu'on fait on » distribue la décision de planification de l'organisation de la 
séance. Le second «on » propose une co-élaboration de l'énoncé que le président va reprendre pour construire sa réponse. L'énoncé du président est fortement incorporé. Le pointage sur l'avocat coproduit avec l'adverbe « déjà » opère une complétion non verbale de l'énoncé du président (Streeck et al., 2011). L'adverbe « déjà » marque une orientation vers la clôture de l'interruption. L'absence d'utilisation d'un microphone et la direction du regard (1.41) confirme que le président s'adresse au juge assesseur et non pas au site distant, maintenant ainsi la distribution de l'activité.

Cette complétion non-verbale permet au juge assesseur de reprendre la parole avant même que le président termine son tour (1.42-43). Cette reprise du nom de l'avocat atteste de la complétude du tour du président. L'utilisation d'un microphone confère au juge assesseur le droit et l'obligation de rendre compte de ce qui vient d'être dit. Le président continue son tour en chevauchement (1.42) avec le juge assesseur en sélectionnant par le regard l'avocat: «et on lui donnera bien la parole après ». Par ce changement corporel, le président rend publique l'organisation de l'audience en train de se faire et cherche le soutien de l'avocat. Néanmoins, cette partie du tour ne semble pas avoir été entendue par le juge assesseur engagé dans la répétition de l'énoncé. En effet, le juge assesseur projette par « et ensuite » (1.45) la complétion de l'organisation de la séance. Les marques d'hésitations (1.45) et l'utilisation d'un énoncé syntaxiquement inachevé «nous continuerons avec » (1.47) rendent visible que d'une part le juge assesseur n'a pas entendu le président et d'autre part qu'il ne s'engage pas dans une prise de décision du ressort du président. Le juge assesseur marque la fin de son tour en éteignant et baissant son microphone (1.48). L'acquiescement du prévenu n'est pas produit immédiatement après la fin du tour de JG, mais après que le juge ait ostensiblement posé son microphone.

Le président s'oriente vers la clôture de l'interruption en donnant la parole à l'avocat (1.51) qui commence immédiatement son tour (1.51) en chevauchement avec le président. Les gestes du président (1.51) complètent l'énoncé «puis après » (1.51) tout en favorisant l'alternance de la parole. L'organisation de l'audience ne semble pas définitive, mais renvoyée à une décision ultérieure.

\section{Conclusions}

L'introduction «d'écrans connectés » dans la salle d'audience complexifie l'organisation et l'écologie interactionnelle des salles d'audience. Les juges se doivent de gérer non seulement le déroulement de l'audience, mais aussi prendre en compte ces nouveaux droits et obligations liées aux apparitions de membres distants. Il apparaît nécessaire de documenter les pratiques liées à l'usage de la visioconférence dans les tribunaux.

L'analyse de ce cas singulier révèle les attentes normatives liées à la production d'un appel visiophonique. La forme même de la mise en relation avec le site distant, celle de l'appel, constitue une pré-séquence qui projette d'autres actions. La pré-séquence a des implications séquentielles, mais aussi en termes de cadre de participation. Elle projette la disponibilité de celui qui produit la pré-séquence et le maintien de cette disponibilité. La prise de parole du surveillant en pleine plaidoirie atteste cette disponibilité projetée par l'appel. Ainsi, le choix de la cour d'initier les appels ne fait que déplacer le problème d'interruption. La cour peut maintenir un contrôle plus accru sur le déroulement de l'audience, mais ne se met pas à l'abri de toute interruption.

Nous avons pu montrer que l'apparition à l'écran est précédée d'un travail préparatoire multimodal de l'appelant pour traiter cet appel. L'apparition à l'image du site distant n'est pas traitée immédiatement. La production de la réponse est retardée par l'ajustement multimodal du dispositif qui retarde la production d'une réponse. Le juge assesseur ajuste le cadrage de la caméra pour mettre la cour à l'écran avant de prendre la parole. Ce travail préparatoire répond à des attentes normatives propres à la visiocommunication (de Fornel, 1994) et accentue le caractère disruptif de cette apparition.

Le traitement de cette apparition requiert un alignement des participants et notamment du locuteur en action. La résistance de l'avocat pour suspendre sa plaidoirie atteste d'une tension entre les attentes normatives de l'appel visiophonique et celles du déroulement attendu d'une audience. Les «écrans 
connectés » rendent ainsi vulnérable l'organisation séquentielle des pratiques interactionnelles en contexte institutionnel.

\section{Références bibliographiques}

Atkinson, J.M. \& Drew, P. (1979). Order in court, Atlantic Highlands: Oxford Socio-Legal Studies.

Boden, D. (1995). Agendas and arrangements: Everyday negotiations in meetings. In A. Firth (Ed.), The discourse of Negotiation Studies of Language in the Worplace (83-99). Pergamon, Elsevier Science.

Bonu, B., (2006). L'interaction visiophonique dans son contexte: le cas d'un "aparté silencieux. Verbum: La pertinence du contexte : contributions de l'ethnométhodologie et de l'analyse conversationnelle, 26(2 - 3), 257278.

Bonu, B. (in press). L'interaction de part et d'autre des barreaux : catégorisations dans la vidéocommunication. Faits de langue.

Couper-Kuhlen, E., \& Thompson, S. A. (2000). Concessive patterns in conversation. In E. C. Traugott, B. Kortmann, \& E. Couper-Kuhlen (Eds.), Cause - Condition - Concession - Contrast. Berlin, New York : Mouton de Gruyter, $381-410$.

Deppermann, A., Schmitt, R., \& Mondada, L. (2010). Agenda and emergence: Contingent and planned activities in a meeting. Journal of Pragmatics, 42(4), 1700-1718.

De Fornel, M. (1994). Le cadre interactionel de l'échange visiophonique. Réseaux, 12(64), 107-132.

Jefferson, G., (2004). Glossary of transcript symbols with an Introduction. In G. H. Lerner, ed. Conversation Analysis: Studies from the first generation. Philadelphia: John Benjamins Publishing Company, 13-23.

Goodwin, C. (1979). The Interactive Construction of a Sentence in Natural Conversation. In G. Psathas (Ed.), Everyday Language: Studies in Ethnomethodology (97-121). New York : Irvington Publishers.

Gumperz, J. J. (1982). Discourse Strategies. Cambridge: Cambridge University Press.

Koschmann, T., LeBaron, C., Goodwin, C., \& Feltovich, P. (2011). “Can you see the cystic artery yet?” A simple matter of trust. Journal of Pragmatics, 43(2), 521-541.

Lerner, G. (2003). "Selecting Next Speaker : The Context-Sensitive Operation of a Context-Free Organization " Language in Society 32(2), 177-201

Licoppe, C. (2006). Ouvrir, suspendre et lever une audience à distance tenue par visioconférence. Études de communication, 29.

Licoppe, C., \& Dumoulin, L. (2007). L'ouverture des procès à distance par visioconférence. Réseaux, 144(5), 103140.

Licoppe, C., \& Dumoulin, L. (2010). The "Curious Case" of an Unspoken Opening Speech Act: A VideoEthnography of the Use of Video Communication in Courtroom Activities. Research on Language \& Social Interaction, 43(3), 211-231.

Licoppe, C., \& Tuncer, S. (in press). Attending to a summons and putting other activities "on hold" Multiactivity as a recognisable interactional accomplishment. In P. Haddington, T. Keisanen, L. Mondada, \& M. Nevile (Eds.), Beyond multitasking: Multiactivity in Social Interaction. John Benjamins Publishing Company.

Mondada, L. (2004). Téléchirurgie et nouvelles pratiques professionnelles : les enjeux interactionnels d'opérations chirurgicales réalisées par visioconférence. Sciences sociales et santé, 22(1), 95-126.

Mondada, L. (2007). Imbrications de la technologie et de l'ordre interactionnel. Réseaux, 144(5), 141-182.

Pomerantz, A. (1984). Agreeing and disagreeing with assessments: some features of preferred/dispreferred turn shapes. In J. M. Atkinson \& J. Heritage (Eds.), Structures of Social Action : Studies in Conversation Analysis (pp. 163-175). Cambridge University Press.

Rintel, S. (2012). Video Calling In Long-Distance Relationships: The Opportunistic Use Of Audio/Video Distortions As A Relational Resource. Electronic Journal Of Communication, 23. 
Sacks, H., (1972). An Initial Investigation of the Usability of Conversational Data for Doing Sociology. In Studies in social interaction. New York: The Free Press, 31-74.

Schegloff, E. A. (1967). The first five seconds: The order of conversational openings. University of California, Berkeley.

Schegloff, E.A. (1968). Sequencing in Conversational Openings. American Anthropologist, 70(6), 1075-1095.

Schegloff, E.A. (1986). The routine as achievement. Human Studies, 9, 111-151.

Schegloff, E. A. (1987). Analyzing Single Episodes of Interaction: An Exercise in Conversation Analysis. Social Psychology Quarterly, 50(2), 101-114.

Schegloff, E.A. \& Sacks, H. (1973). Opening up closings. Semiotica, 8, 289-327.

Streeck, J., Goodwin, C. \& LeBaron, C. (2011). Embodied Interaction language and body in the material world. In J. Streeck, C. Goodwin, \& C. LeBaron, eds. Embodied Interaction language and body in the material world. Cambridge University Press, $1-28$.

Turner, R., (1972). Some formal properties of therapy talk. In D. Sudnow, ed. Studies in Social Interaction. Free Press, 367-396.

Veyrier, C.-A. (2012). Les cinq premières minutes : organisation des ouvertures en (web)conférence, Analyse de pratiques interactionnelles en réunion professionnelle. Thèse de doctorat. Université Montpellier 3. Accessible en ligne : http://tel.archives-ouvertes.fr/tel-00766288

Whalen, J., Zimmerman, D. H., \& Whalen, M. R. (1988). When Words Fail: A Single Case Analysis. Social Problems, 35(4), 335-362.

\section{Conventions de transcription}

Chevauchements

(.)

Micro-pause

Pauses en secondes

/

Intonation montante/ descendante $\backslash$

${ }^{\circ}$ bon $^{\circ}$

Segment produit moins fort

((rire))

Phénomènes non transcrits

:

Allongement vocalique

immédiat-

Troncation

$\&$

Continuation du tour de parole

$=$

Enchaînement rapide

$\mathrm{xxx}$

Segment incompréhensible

(il va)

Transcription incertaine

Les lignes de transcription multimodales ne sont pas numérotées.

$\S \mathrm{PJ}$

$>>$

$<<<$

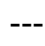

--regard--
Phénomène non sonore attribué à un participant

Mouvement de caméra vers la droite

Mouvement de caméra vers la gauche

Mouvement de caméra vers le haut

Indique le début d'un phénomène décrit

Description du phénomène 
-----> Continuation du phénomène aux lignes suivantes

->---- Continuation d'un phénomène précédent

----| Fin d'un phénomène 